\title{
Congenital Zika Virus Infection in a Birth Cohort in Vietnam, 2017-2018
}

\author{
Mya Myat Ngwe Tun, ${ }^{1}$ Masako Moriuchi, ${ }^{2}$ Michiko Toizumi, ${ }^{3}$ Elizabeth Luvai, ${ }^{1}$ Sandra Raini, ${ }^{1}$ Noriko Kitamura, ${ }^{3}$ Mizuki Takegata, ${ }^{3}$ \\ Hien-Anh Thi Nguyen, ${ }^{4}$ Meng Ling Moi, ${ }^{1}$ Corazon C. Buerano, ${ }^{5}$ Dang Duc Anh, ${ }^{4}$ Lay-Myint Yoshida, ${ }^{3}$ Kouichi Morita, ${ }^{1}$ and \\ Hiroyuki Moriuchi ${ }^{2 *}$ \\ ${ }^{1}$ Department of Virology, Institute of Tropical Medicine and Leading Program, Nagasaki University, Nagasaki, Japan; ${ }^{2}$ Department of Pediatrics, \\ Graduate School of Biomedical Sciences, Nagasaki University, Nagasaki, Japan; ${ }^{3}$ Department of Pediatric Infectious Diseases, Institute of Tropical \\ Medicine, Nagasaki University, Nagasaki, Japan; ${ }^{4}$ National Institute of Hygiene and Epidemiology, Hanoi, Vietnam; ${ }^{5}$ Research and Biotechnology, \\ St. Luke's Medical Center, Quezon City, Philippines
}

\begin{abstract}
To detect congenital ZIKV infection (CZI) in a birth cohort and among high-risk neonates in Vietnam, we collected umbilical cord blood plasma samples of newly delivered babies and peripheral plasma samples of high-risk neonates in Nha Trang, central Vietnam, between July 2017 and September 2018. Samples were subjected to serological and molecular tests. Of the 2013 newly delivered babies, 21 (1\%) were positive for Zika virus (ZIKV) IgM and 1,599 (79\%) for Flavivirus IgG. Among the 21 ZlKV IgM-positives, 11 were confirmed to have CZI because their plasma samples had anti-ZIKV neutralization titers $\geq 4$ times higher than those against dengue virus (DENV)- 1 to 4 and Japanese encephalitis virus (JEV) and were tested for the ZIKV RNA positive by real-time reverse transcription-PCR. Therefore, the incidence of $\mathrm{CZI}$ in our birth cohort was approximately $0.5 \%$. Of the 150 high-risk neonates, three (2\%) and $95(63 \%)$ were positive for ZIKV IgM and Flavivirus IgG antibodies, respectively. None of the three ZIKV IgM-positives had $\geq 4$ times higher anti-ZIKV neutralization titers than those against DENV-1 to 4 and JEV, and were therefore considered as probable CZI. Our results indicate that CZI is not rare in Vietnam. Although those with confirmed CZI did not show apparent symptoms suspected of congenital Zika syndrome at birth, detailed examinations and follow-up studies are needed to clarify the CZI impact in Vietnam. This is the first report of CZI cases in a birth cohort in Asia.
\end{abstract}

\section{INTRODUCTION}

Zika virus (ZIKV) of the family Flaviviridae, genus Flavivirus can be transmitted to humans through the vector Aedes mosquitoes or through nonvector transmission such as sexual contact, maternal-fetal transmission, and blood transfusions. ${ }^{1-5}$ The first human case of ZIKV infection was reported in 1954 in Nigeria, and sporadic cases have been noted in Asia. ${ }^{6,7}$ It has been widely reported that approximately $80 \%$ of people with ZIKV infection are asymptomatic. ${ }^{8,9}$ Although the disease is self-limiting, cases of neurological manifestations have been described. Between 2015 and 2016, ZIKV had been of global health concern following large outbreaks in the Americas and the observed associated congenital abnormalities, including microcephaly, intrauterine growth restriction, blindness, and stillbirth. ${ }^{10}$ Despite a long period of ZIKV circulation in Asia, only three confirmed cases of congenital ZIKV infection (CZI) with microcephaly were reported in this region: two in Thailand and one in Vietnam. ${ }^{11,12}$ In Vietnam, 219 and 13 cases of ZIKV disease were reported in 2016 and 2017 (JanuaryFebruary), respectively. ${ }^{13}$ No data are available on the incidence and embryotoxicity of CZI in a birth cohort in Asia. We herein report data of ZIKV infection from 1) a large-scale birth cohort study on mother-to-child infections and 2) investigation of neonates who were suspected with congenital infection in Vietnam.

\section{MATERIALS AND METHODS}

Study participants and sample collection. The present study was conducted in Khanh Hoa General Hospital (KHGH), Nha Trang, Vietnam, from July 2017 to September 2018, and

\footnotetext{
*Address correspondence to Hiroyuki Moriuchi, Department of Pediatrics, Graduate School of Biomedical Sciences, Nagasaki University, 1-7-1 Sakamoto, Nagasaki 852-8501, Japan. E-mail: hiromori@nagasaki-u.ac.jp
}

consisted of two parts. For the first part of the study, we enrolled all women who 1) delivered their babies at $\mathrm{KHGH}, 2$ ) were 18 years or older at the time of delivery, and 3) resided in selected 16 communes in Nha Trang, during the study period. Exclusion criteria for this part of the study were women who had 1) spontaneous/induced abortions or stillbirths, 2) multiple pregnancies, or 3 ) serious complication from/during this pregnancy. Blood samples were collected from umbilical cords of babies just after their delivery at the obstetrics ward. ethylenediaminetetraacetic acid-treated tubes were used for blood collection. Plasma was separated by centrifugation $(3,000 \mathrm{rpm} \times 10$ minutes $)$ and kept in $\mathrm{a}-80^{\circ} \mathrm{C}$ freezer until testing.

For the second part of the study, during the study period, we enrolled high-risk neonates (children aged 28 days or less) 1) born at $\mathrm{KHGH}$ from women who had any two disease symptoms such as fever, rash, arthralgia/arthritis, lymphadenopathy, and conjunctivitis, or 2) born at $\mathrm{KHGH}$ or referred to neonate intensive care unit/pediatric department in $\mathrm{KHGH}$ and who had any symptoms related to congenital infection such as suspected meningoencephalitis, microcephaly, hydrocephalus, glaucoma, cataract, thrombocytopenia, purpura, hearing impairment, and lymphadenopathy, or who had head circumference of $<30 \mathrm{~cm}$ at birth, and whose birth weight for gestation age was equal or below the cutoff on birth weight patterns by gestation age reference setting. ${ }^{14,15}$ Exclusion criteria for the second part were neonates with confirmed chromosomal abnormality or those with well-known congenital syndrome related to a genetic defect. Some of the participants in the second part of the study could also be included in the first part if their mothers lived in the catchment area and delivered them at $\mathrm{KHGH}$ during the study period. Peripheral blood samples were collected from the neonates, and the plasma was separated and kept as earlier.

Written informed consents were obtained from mothers for their participation in the first part of the study as well as for their 
babies' participation in the first and/or second parts of the study.

This work was approved by the Ethics Committees of the Institute of Tropical Medicine, Nagasaki University, Japan (160908158), and the National Institute of Hygiene and Epidemiology, Hanoi, Vietnam (IRB-VN01057-30/2015).

Viruses and cell lines. The virus strains used for the serological tests, namely, IgM capture ELISA, Flavi IgG ELISA, and neutralization tests, were as follows: MR 766 (ZIKV), 99St12A (dengue virus [DENV]-1), 00st22A (DENV-2), SLMC50 (DENV3), SLMC318 (DENV-4), and JaOrS982 (Japanese encephalitis virus [JEV]). These viruses were propagated in C6/36 Aedes albopictus mosquito cells and were used to inoculate the Vero (African green monkey kidney epithelial cell line ATCC, CCL81) cell line for virus titration and neutralization tests.

IgM capture ELISAs for the detection of ZIKV, DENV, and JEV infections. In-house IgM capture ELISAs were carried out using the protocol described previously with minor modifications. ${ }^{16}$ Each well of 96-well microplates (Maxisorp, Nalge Nunc International, Roskilde, Denmark) was coated with $100 \mu \mathrm{L}(5.5 \mu \mathrm{g} / 100 \mu \mathrm{L})$ of antihuman IgM goat IgG antibody (Cappel ICN Pharmaceuticals, Aurora, OH) in ELISA coating buffer (0.05 M carbonate-bicarbonate buffer, $\mathrm{pH} 9.6$, containing $0.0 \%$ sodium azide). Plates were then incubated at $37^{\circ} \mathrm{C}$ for 1 hour or at $4^{\circ} \mathrm{C}$ overnight. Each well was blocked with $100 \mu \mathrm{L}$ of BlockAce (UK-1 B 80, Yukijirushi, Sapporo, Japan), except for the blank wells, and plates were incubated at room temperature for 1 hour. After incubation, wells were washed three times with phosphate-buffered saline (PBS) without calcium and magnesium but containing $0.1 \%$ Tween 20 (PBSTween 20 [PBS-T]). Test samples, as well as positive and negative control samples, were diluted at 1:100 in PBS-T, and $100 \mu \mathrm{L}$ aliquots of these samples were distributed into duplicate wells. Plates were incubated at $37^{\circ} \mathrm{C}$ for 1 hour and then washed as described earlier. Zika virus or tetravalent DENV or JEV antigen (128 ELISA units) at $100 \mu \mathrm{L} /$ well was added after which the plates were incubated at $37^{\circ} \mathrm{C}$ for 1 hour. After washing as described earlier, horseradish peroxidase (HRP)-conjugated anti-Flavivirus mouse monoclonal antibody (12D11/7E8) at 1:1,000 dilution for anti-ZIKV IgM capture ELISA, or 1:1,500 dilution for anti-DENV or anti-JEV IgM capture ELISA was added at $100 \mu \mathrm{L} /$ well. Plates were incubated at $37^{\circ} \mathrm{C}$ for 1 hour and washed as earlier. Color was developed by adding in each well a $100-\mu \mathrm{L}$ volume of $5 \mathrm{mg}$ o-phenylenediamine dihydrochloride (OPD) (Sigma Chemical, St. Louis, MO) with $0.0 \%$ hydrogen peroxide in $10 \mathrm{~mL}$ of $0.05 \mathrm{M}$ citrate phosphate buffer, $\mathrm{pH}$ 5.0. Plates were kept at room temperature for 30-60 minutes in a dark place. To terminate the reaction, $100 \mu \mathrm{L}$ of $1 \mathrm{~N}$ sulfuric acid was added to each well, and then the optical density (OD) was read at $492 \mathrm{~nm}$ (Multiscan JX, model no. 353; Thermolab System, Tokyo, Japan). A positive control (or test sample)/ negative control OD ratio greater than or equal to 2.0 was considered positive.

Anti-Flavivirus IgG ELISA. In-house indirect IgG ELISA was performed to detect the presence of anti-Flavivirus IgG in plasma samples, and a purified JEV was used as assay antigen. ${ }^{17}$ In the procedure, all wells of the 96-well microplates, except for the blanks, were coated with $100 \mu \mathrm{L}$ of JEV antigen (250 ng/100 $\mu \mathrm{L} /$ well) diluted with ELISA-coating buffer. Plates were incubated at $37^{\circ} \mathrm{C}$ for 1 hour or at $4^{\circ} \mathrm{C}$ overnight. All wells except for the blanks were blocked with $100 \mu \mathrm{L}$ of the original concentration of BlockAce and were incubated at room temperature for 1 hour. Plates were washed three times with PBS-T, after which $100 \mu \mathrm{L}$ of each test plasma sample diluted at 1:1,000 in PBS-T + 10\% BlockAce was added in duplicate wells in each plate. Control sample known to contain the antibody to test antigen was run on each plate as a positive control. After incubation at $37^{\circ} \mathrm{C}$ for 1 hour, plates were washed, and 1:30,000 diluted HRP-conjugated antihuman IgG goat IgG (American Qualex, San Clemente, CA) in PBS-T $+10 \%$ BlockAce was added at $100 \mu \mathrm{L} /$ well. Plates were incubated at $37^{\circ} \mathrm{C}$ for 1 hour, followed by washing. Initiation of the peroxidase reaction was performed by the addition of OPD substrate solution (described earlier) at $100 \mu \mathrm{L} /$ well. Plates were incubated at room temperature for 30-60 minutes in the dark, and then the reaction was stopped by the addition of $1 \mathrm{~N}$ sulfuric acid at 100 $\mu \mathrm{L} /$ well. A standard curve was prepared by using the $\mathrm{OD}_{492}$ values of the positive control serum starting with a 1,000-fold dilution, followed by serial 2-fold dilutions up to $1: 2^{12}$ in PBS-T + $10 \%$ BlockAce. IgG titers of test serum samples were determined from the positive standard curve. A sample titer equal to or greater than 1:3,000 was considered to be positive.

Focus reduction neutralization test. To confirm the status of ZIKV infection in the study subjects, plasma samples were checked for the ability to neutralize ZIKV, the four serotypes of DENV, and JEV by $50 \%$ focus reduction neutralization test (FRNT ${ }_{50}$ ). ${ }^{16,18}$ Plasma samples were heat-treated at $56^{\circ} \mathrm{C}$ for 30 minutes and diluted serially. Serially diluted samples at $150-\mu \mathrm{L}$ volumes were mixed with equal volumes of specific virus at 60 focus-forming units, and mixtures were incubated at $37^{\circ} \mathrm{C}$ for 1 hour for virus-antibody neutralization reaction. Each mixture was inoculated onto Vero cell monolayer in 96well plates. After incubation at $37^{\circ} \mathrm{C}$ for 1 hour, the infected cells were overlaid with $1.3 \%$ methylcellulose 4,000 in $2 \%$ fetal calf serum minimum essential media. The plates with ZIKV or JEV were then incubated at $37^{\circ} \mathrm{C}$ for 2 days, and the plates with DENV were incubated for 3 days in the same temperature. The plates were washed with PBS (-). Cells in each plate were fixed with $4 \%$ paraformaldehyde phosphatebuffered solution for 30 minutes at room temperature, rinsed, and were permeabilized with 1\% NP-40 solution in PBS (-) for 30 minutes at room temperature. After washing, the plates were blocked with BlockAce for 30 minutes at room temperature. Pooled human serum samples with a high titer of antiFlavivirus IgG (diluted 1:1,500) were then added per well; plates were then incubated at $37^{\circ} \mathrm{C}$ for 1 hour and washed. Subsequently, 1:1,000 diluted HRP-conjugated goat antihuman IgG was added to each well, after which plates were incubated at $37^{\circ} \mathrm{C}$ for 1 hour. Staining of positive cells was visualized by the addition of a $0.5-\mathrm{mg} / \mathrm{mL}$ solution of substrate 3, 3'-diaminobenzidine tetrahydrochloride in PBS (-) with $0.0 \%$ of $\mathrm{H}_{2} \mathrm{O}_{2}$ at room temperature. Staining reaction was allowed to proceed for 10 minutes, after which the cells were washed. The number of foci of stained cells per well was counted under a microscope. The reciprocal of the endpoint serum dilution that provided a $50 \%$ or greater reduction in the mean number of foci relative to the control wells that contained no serum was considered to be the $\mathrm{FRNT}_{50}$ titer. The volume used for all the reactants in this test was at $100 \mu \mathrm{L} /$ well.

Conventional and real-time reverse transcription-PCR (qRT-PCR) for ZIKV. Viral RNA was directly extracted from plasma by using Viral RNA Mini Kit (QIAGEN, Hilden, Germany) according to the manufacturer's instructions. The qRT-PCR was performed by using TaqMan Fast Virus 1-Step Master Mix 
kit (Applied Biosystems, Foster City, CA) following the protocol from a previous report with three primer sets for ZIKVE and NS5 genes. ${ }^{19-23}$ Cycle threshold value $<40$ was considered as ZIKV positive for qRT-PCR. Standard complementary DNA at 10 -fold serial dilutions $\left(10^{8}-10^{2}\right.$ genome copies) was applied for quantification of viral genome levels. ${ }^{20}$ The viral genome levels were expressed as $\log _{10}$ genome copies $/ \mathrm{mL}$. Conventional RT-PCR was performed by using Prime Script one step RT-PCR Kit (Takara Bio Inc., Shiga, Japan) following the manufacturer's instructions and using previously published primers for ZIKVNS3 gene. ${ }^{24}$

Zika virus case classification. We regarded a case as CZI if ZIKV RNA was detected in the cord or neonatal blood plasma or if the neonate was ZIKV IgM positive and positive for neutralization test only against ZIKV but not with other flaviviruses or when the neutralizing antibody titer against ZIKV was $\geq 4$ times higher than the antibody titers against other flaviviruses. This definition is consistent with confirmed ZIKV infection in the WHO criteria. ${ }^{25}$ The WHO defined a ZIKV infection as a probable case if the clinical sample is positive for IgM antibody against ZIKV and the ratio of ZIKV neutralization titer to other Flavivirus neutralization titers is less than 4 and with no ZIKV RNA detected by RT-PCR. ${ }^{25}$ In case there is no adequate plasma sample for the performance of RT-PCR, we also considered infection as probable. In this study, we called our probable case as probable $\mathrm{CZI}$ and regarded it as nonconfirmed CZI.

\section{RESULTS}

In total, 2015 mothers, who were about to give birth, were enrolled in the first part of the study, and a total of 2013 umbilical cord plasma samples from their newly born babies were analyzed. In the second part of the study, 150 neonates were enrolled, and their plasma samples were also analyzed. In the first part of the study, 21 (1\%) of the 2013 newly born babies were positive for ZIKV IgM, seven (0.3\%) for DENV IgM, and 1,597 (79.3\%) for Flavivirus IgG (Table 1). Among the 21 ZIKV IgM-positives, nine were positive for JEV and/or DENV IgM. In the second part of the study, of the 150 neonates, three $(2 \%)$, two (1.3\%), and 95 (63.3\%) were positive for ZIKV IgM, DENV $\operatorname{lgM}$, and Flavivirus IgG, respectively (Table 1). Of the three ZIKV IgM-positives, one was JEV IgM positive.

Among the 21 ZIKV IgM-positive newly born babies, 11 had anti-ZIKV neutralization titers $\geq 4$ times higher the neutralization titers against DENV-1 to 4 and JEV (Table 2). Therefore, 11 $(0.5 \%)$ in the birth cohort were confirmed as CZI. In addition, their samples had ZIKV IgM $P / N$ ratios higher than those for DENV and JEV. Six of the 11 babies had plasma ZIKV RNAs that were positive in the real-time PCR in the two primer sets used in the study, whereas five were positive in one of the two primer sets. However, detection of their ZIKV RNAs by conventional RT-PCR showed negative results. The other 10 of 21
ZIKV IgM-positives had cord blood plasma samples with antiZIKV neutralization titers $<4$ times than those against DENV-1 to 4 and JEV, and thus were considered to have probable CZI.

The three ZIKV IgM-positive neonates in the second part of the study had plasma samples with anti-ZIKV neutralization titers $<4$ times than those against DENV-1 to 4 and JEV. They were considered as probable CZI and hence were not confirmed CZI. With regard to annual and seasonal patterns for $\mathrm{CZI}$, there were nine confirmed case of CZI in July-November 2017 and two in July-September 2018, suggesting annual variability and seasonality. Our findings showed approximately $0.5 \%$ incidence of CZI, or 5.5 per 1,000 live births in our birth cohort study in central Vietnam.

Among the 11 confirmed CZI cases, nine babies did not show any apparent symptoms at birth and were discharged together with their mothers after 5 days as usual delivery hospitalization. One baby girl who showed symptoms of illness vomited repeatedly and did not suck her mother's breast milk on the next day after birth. Her white blood cell count was $20,110 / \mu \mathrm{L}$ (neutrophil $67.7 \%$ ). She was hospitalized in the pediatric ward, treated with antibiotics for neonatal infection, and given phototherapy for neonatal jaundice. She was discharged after 5 days of hospitalization. The other baby girl who showed symptoms of illness was born to a mother who had pre-labor rupture of membranes. The baby, too, had vomited and could not be breastfed. Her white blood cell count was $28,300 / \mu \mathrm{L}$ (neutrophil $71.0 \%$ ) on the day of birth. She was treated with antibiotics for neonatal bacterial infection and had phototherapy for neonatal jaundice at the pediatric department for 11 days. After getting well, she was discharged. These two baby girls did not show any symptoms suspected as congenital infection as listed in the inclusion criteria for the second part of the study, and they were not enrolled in the second part. The 10 cases with probable CZI in the first part of the study had no apparent symptoms at birth.

\section{DISCUSSION}

In this study, we reported the occurrence of CZI among babies delivered in Nha Trang, Vietnam. Their infections were confirmed based on the positive detection of ZIKV RNA, IgM against ZIKV, and neutralizing antibodies against ZIKV by using their cord blood plasma samples. It was noted that these samples had positive results for IgM and/or neutralization activity against more than one virus. This could be because of cross-reaction of the tested antibodies against ZIKV, DENV, and JEV, all of which belong to the same Flaviviridae family. ${ }^{26-28}$ High percentage of cord blood and neonate plasma samples positive for anti-Flavivirus IgG was observed, and it could be because of the passively transferred maternal IgG. ${ }^{29}$ Increasing number of divergent ZIKV strains that highlight genetic variability is regarded as a potential limiting factor

TABLE 1

Positive rates of ZIKV IgM, DENV IgM, and Flavivirus IgG in pregnant women and neonates, Nha Trang, Vietnam, July 2017-September 2018

\begin{tabular}{lcrrr}
\hline \multicolumn{1}{c}{ Source of samples } & Total no. of samples & ELISA-positive samples \\
\cline { 3 - 5 } & 2,013 & $21(1.0 \%)$ & DENV IgM & $7(0.3 \%)$ \\
\hline Cord blood plasma in the birth cohort & 150 & $3(2.0 \%)$ & $2(1.3 \%)$ & $1,597(79.3 \%)$ \\
Plasma from high-risk neonates & & & $95(63.3 \%)$ \\
\hline DENV = dengue virus; ZIKV = Zika virus. & &
\end{tabular}




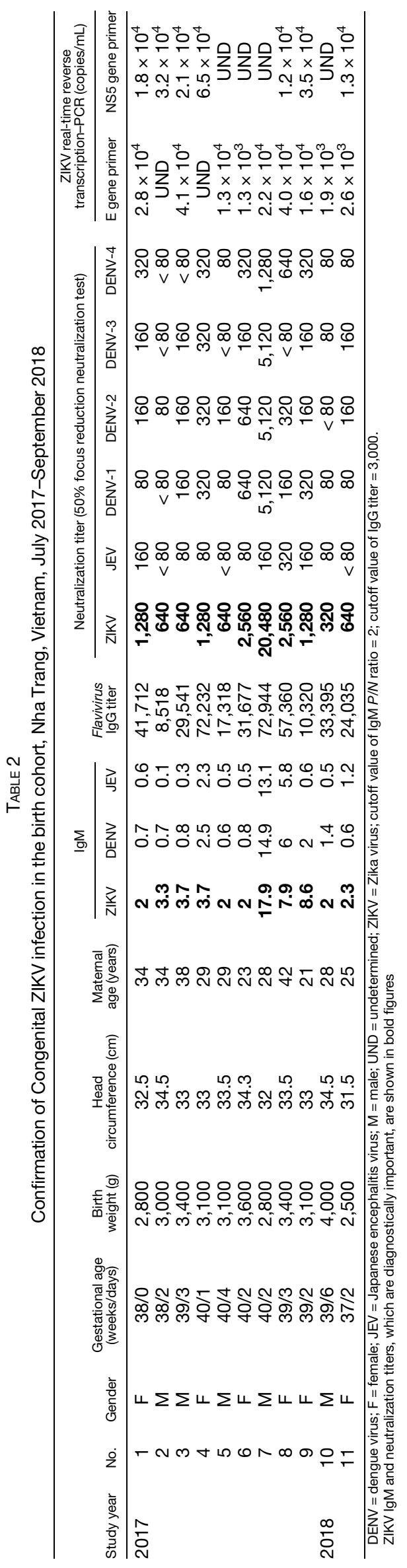

of the sensitivity of ZIKV qRT-PCR based diagnosis; therefore, a previous study suggested to use several QRT-PCR targets for diagnosis. ${ }^{23}$ The 11 babies with confirmed CZI in this study had their plasma samples positive for QRT-PCR in at least one primer set. However, conventional PCR performed to plasma positives by qRT-PCR showed negative results which could be because of low viral loads in the plasma. Previous studies reported that conventional RT-PCR could detect ZIKV viral load in most of the samples, with an estimated $10^{6}-10^{10} \mathrm{RNA}$ copies $/ \mathrm{mL}^{23,30}$

Published studies showed strong association between microcephaly and ZIKV infection confirmed by qRT-PCR, capture IgM ELISA, or both. ${ }^{21,31,32}$ In Brazil, 32 of 91 neonates born with microcephaly were confirmed positive for ZIKV infection by qRT-PCR or anti-ZIKV IgM ELISA with confirmation more frequent in cerebrospinal fluid than in serum. ${ }^{33}$ Also in Brazil, another study reported that levels of ZIKV IgM and neutralizing antibodies were higher in babies with microcephaly cases than in the neonate controls (at the time of birth) and their mothers. ${ }^{34}$ In our study, we confirmed CZI in $0.5 \%$ (11/2013) of newly born babies based on the results of IgM capture ELISA, qRT-PCR, and neutralization test by using their cord blood samples. Comparing our data from the Americas where the rate of microcephaly/CZI varies from $5 \%$ to $14 \%,{ }^{35,36}$ our study showed a low rate of CZI infection, and it could be associated with the differences in the characteristics of the ZIKV belonging to the different clades of Asian lineage. A previous study on the importation of ZIKV from Vietnam to Japan in 2016 indicated that the isolated virus belonged to the Southeast Asian clade of the Asian lineage, and it was distinct from the ZIKV isolates (American clade of Asian lineage) in the Americas. ${ }^{37}$ Other reports suggested that Southeast Asian clade of ZIKV had lower replicative ability than the American clade of Asian lineage/African lineage. ${ }^{38}$

This is the first report of ZIKV infection in a birth cohort in Asia. Our results indicate that $\mathrm{CZI}$ is not rare in Vietnam. Even though eye examinations and brain imaging were not conducted in this study, all the infected babies did not show apparent symptoms suspected of congenital Zika syndrome, which are characterized by severe microcephaly (in which the skull has partially collapsed), decreased brain tissue with a specific pattern of brain damage, damage to the back of the eye, congenital contractures, and hypertonia restricting body movement soon after birth. ${ }^{39,40}$ Newborns whose mothers are infected with ZIKV during pregnancy have a $5-14 \%$ risk of congenital Zika syndrome and a 4-6\% risk of ZIKV-associated microcephaly, ${ }^{35,36,41-46}$ whereas a study involving pregnant women from Rio de Janeiro used a broader definition for ZIKVassociated outcomes and identified adverse outcomes in $42 \%$ of fetuses and infants exposed to the virus. ${ }^{41}$ Thus, generally, the number of children who were born to mothers with ZIKV infection during pregnancy but who did not have apparent disability at birth is large, and our findings are in agreement with that. However, a previous study found that infants with in utero ZIKV exposure without congenital Zika syndrome appeared at risk for abnormal neurodevelopmental outcomes in the first 18 months of life. ${ }^{39}$ Therefore, detailed clinical assessment combined with ophthalmologic examination, hearing screening, and brain imaging, and long-term follow-up including neurodevelopmental surveillance of infected offspring are needed to clarify the impact of CZI in Vietnam. 
Received April 13, 2020. Accepted for publication June 26, 2020.

Published online August 17, 2020.

Acknowledgments: We would like to thank the staff in Vietnam who supported the study.

Financial support: This work was supported by Grants-in-Aid for Scientific Research (Kakenhi, 16H05846), Japan Initiative for Global Research Network on Infectious Diseases (JGRID, JP19fm0108001), Japan-U.S. Cooperative Medical Science Program from the Japan Agency for Medical Research and Development (AMED, 19fk0108035h1203), and Joint Usage/Research Center on Tropical Disease, Institute of Tropical Medicine, Nagasaki University.

Authors' addresses: Mya Myat Ngwe Tun, Elizabeth Luvai, Sandra Raini, Meng Ling Moi, and Kouichi Morita, Department of Virology, Institute of Tropical Medicine and Leading Program, Nagasaki University, Nagasaki, Japan, E-mails: myamyat@tm.nagasaki-u.ac.jp, ajemaluva@gmail.com, kendraraini@gmail.com, sherry@nagasakiu.ac.jp, and moritak@nagasaki-u.ac.jp. Masako Moriuchi and Hiroyuki Moriuchi, Department of Pediatrics, Graduate School of Biomedical Sciences, Nagasaki University, Nagasaki, Japan, E-mails: a65-masa@nagasaki-u.ac.jp and hiromori@nagasaki-u.ac.jp. Michiko Toizumi, Noriko Kitamura, Mizuki Takegata, and Lay-Myint Yoshida, Department of Pediatric Infectious Diseases, Institute of Tropical Medicine, Nagasaki University, Nagasaki, Japan, E-mails: toizumi@ nagasaki-u.ac.jp, norikok0110@gmail.com, mtakegata-tky@umin.ac.jp, and Imyoshi@nagasaki-u.ac.jp. Hien-Anh Thi Nguyen and Dang Duc Anh, National Institute of Hygiene and Epidemiology, Hanoi, Vietnam, E-mails: hienanh75@yahoo.com and dangducanh.nihe@gmail.com. Corazon C. Buerano, Research and Biotechnology, St. Luke's Medical Center, Quezon City, Philippines, E-mail: ccbuerano@hotmail.com.

This is an open-access article distributed under the terms of the Creative Commons Attribution (CC-BY) License, which permits unrestricted use, distribution, and reproduction in any medium, provided the original author and source are credited.

\section{REFERENCES}

1. Musso D, Gubler DJ, 2016. Zika virus. Clin Microbiol Rev 29: 487-524.

2. Musso D, Stramer SL, Busch MP, 2016. Zika virus: a new challenge for blood transfusion. Lancet 387: 1993-1994.

3. Foy BD, Kobylinski KC, Chilson Foy JL, Blitvich BJ, Travassos da Rosa A, Haddow AD, Lanciotti RS, Tesh RB, 2011. Probable non-vector-borne transmission of Zika virus, Colorado, USA. Emerg Infect Dis 17: 880-882.

4. D'Ortenzio $E$, Matheron S, Yazdanpanah $Y$, de Lamballerie $X$, Hubert B, Piorkowski G, Maquart M, Descamps D, Damond F, Leparc-Goffart I, 2016. Evidence of sexual transmission of Zika virus. N Engl J Med 374: 2195-2198.

5. Calvet $\mathrm{G}$ et al., 2016. Detection and sequencing of Zika virus from amniotic fluid of fetuses with microcephaly in Brazil: a case study. Lancet Infect Dis 16: 653-660.

6. Mlakar $\mathrm{J}$ et al., 2016. Zika virus associated with microcephaly. N Engl J Med 374: 951-958.

7. Chang C, Ortiz K, Ansari A, Gershwin ME, 2016. The Zika outbreak of the 21st century. J Autoimmun 68: 1-13.

8. Duffy MR et al., 2009. Zika virus outbreak on Yap Island, Federated States of Micronesia. N Engl J Med 360: 2536-2543.

9. Petersen EE, Staples JE, Meaney-Delman D, Fischer M, Ellington SR, Callaghan WM, Jamieson DJ, 2016. Interim guidelines for pregnant women during a Zika virus outbreak-United States, 2016. MMWR Morb Mortal Wkly Rep 65: 30-33.

10. Desai SK, Hartman SD, Jayarajan S, Liu S, Gallicano GI, 2017. Zika virus (ZIKV): a review of proposed mechanisms of transmission and associated congenital abnormalities. Am J Stem Cells 6: 13-22.

11. Wongsurawat $T$ et al., 2018. Case of microcephaly after congenital infection with Asian lineage Zika virus, Thailand. Emerg Infect Dis 24: 1758-1761.

12. Moi ML et al., 2017. Zika virus infection and microcephaly in Vietnam. Lancet Infect Dis 17: 805-806.

13. Lim SK, Lim JK, Yoon IK, 2017. An update on Zika virus in Asia. Infect Chemother 49: 91-100.
14. Miyakawa M, Yoshino $H$, Yoshida LM, Vynnycky E, Motomura $H$, Tho LH, Thiem VD, Ariyoshi K, Anh DD, Moriuchi H, 2014. Seroprevalence of rubella in the cord blood of pregnant women and congenital rubella incidence in Nha Trang, Vietnam. Vaccine 32: 1192-1198.

15. Mikolajczyk RT, Zhang J, Betran AP, Souza JP, Mori R, Gulmezoglu AM, Merialdi M, 2011. A global reference for fetal-weight and birth weight percentiles. Lancet 377: 1855-1861.

16. Ngwe Tun MM et al., 2013. Serological characterization of dengue virus infections observed among dengue hemorrhagic fever/ dengue shock syndrome cases in upper Myanmar. J Med Virol 85: 1258-1266.

17. Inoue $\mathrm{S}$ et al., 2010. Evaluation of a dengue IgG indirect enzymelinked immunosorbent assay and a Japanese encephalitis IgG indirect enzyme-linked immunosorbent assay for diagnosis of secondary dengue virus infection. Vector Borne Zoonotic Dis 10: $143-150$.

18. Ngwe Tun MM et al., 2018. Detection of Zika virus infection in Myanmar. Am J Trop Med Hyg 98: 868-871.

19. Kyaw AK et al., 2017. Clinical, virological and epidemiological characterization of dengue outbreak in Myanmar, 2015. Epidemiol Infect 145: 1886-1897.

20. Ito M, Takasaki T, Yamada K, Nerome R, Tajima S, Kurane I, 2004. Development and evaluation of fluorogenic TaqMan reverse transcriptase PCR assays for detection of dengue virus types 1 to 4. J Clin Microbiol 42: 5935-5937.

21. Lanciotti RS, Kosoy OL, Laven JJ, Velez JO, Lambert AJ, Johnson AJ, Stanfield SM, Duffy MR, 2008. Genetic and serologic properties of Zika virus associated with an epidemic, Yap state, Micronesia, 2007. Emerg Infect Dis 14: 1232-1239.

22. Wilson HL, Tran T, Druce J, Dupont-Rouzeyrol M, Catton M, 2017. Neutralization assay for Zika and dengue viruses by use of realtime-PCR-based endpoint assessment. J Clin Microbiol 55: 3104-3112.

23. Corman VM et al., 2016. Assay optimization for molecular detection of Zika virus. Bull World Health Organ 94: 880-892.

24. Grard G, Caron M, Mombo IM, Nkoghe D, Mboui Ondo S, Jiolle D, Fontenille D, Paupy C, Leroy EM, 2014. Zika virus in Gabon (Central Africa)-2007: a new threat from Aedes albopictus? PLoS Negl Trop Dis 8: e2681.

25. World Health Organization, 2016. Available at: https://who.int/ health-topics/zika-virus-disease/publications/epidemiologyand-surveillance. Accessed July 31, 2020.

26. Monath TP, Nystrom RR, Bailey RE, Calisher CH, Muth DJ, 1984. Immunoglobulin $\mathrm{M}$ antibody capture enzyme-linked immunosorbent assay for diagnosis of St. Louis encephalitis. J Clin Microbiol 20: 784-790.

27. Lindsey NP et al., 2018. Ability to serologically confirm recent Zika virus infection in areas with varying past incidence of dengue virus infection in the United States and U.S. territories in 2016. J Clin Microbiol 56: e01115-17.

28. Martin DA, Muth DA, Brown T, Johnson AJ, Karabatsos N, Roehrig JT, 2000. Standardization of immunoglobulin M capture enzyme-linked immunosorbent assays for routine diagnosis of arboviral infections. J Clin Microbiol 38: 1823-1826.

29. Singh T et al., 2019. Efficient transplacental IgG transfer in women infected with Zika virus during pregnancy. PLoS Negl Trop Dis 13: e0007648.

30. Paiva MHS, Guedes DRD, Leal WS, Ayres CFJ, 2017. Sensitivity of RT-PCR method in samples shown to be positive for Zika virus by RT-qPCR in vector competence studies. Genet $\mathrm{Mol}$ Biol 40: 597-599.

31. Cordeiro MT, Pena LJ, Brito CA, Gil LH, Marques ET, 2016. Positive IgM for Zika virus in the cerebrospinal fluid of 30 neonates with microcephaly in Brazil. Lancet 387: 1811-1812.

32. Haby MM, Pinart M, Elias V, Reveiz L, 2018. Prevalence of asymptomatic Zika virus infection: a systematic review. Bull World Health Organ 96: 402D-413D.

33. de Araujo TVB et al., 2018. Association between microcephaly, Zika virus infection, and other risk factors in Brazil: final report of a case-control study. Lancet Infect Dis 18: 328-336.

34. Castanha PMS et al., 2019. Perinatal analyses of Zika- and dengue virus-specific neutralizing antibodies: a microcephaly 
case-control study in an area of high dengue endemicity in Brazil. PLoS Negl Trop Dis 13: e0007246.

35. Hoen B et al., 2018. Pregnancy outcomes after ZIKV infection in French territories in the Americas. N Engl J Med 378: 985-994.

36. Shapiro-Mendoza CK et al., 2017. Pregnancy outcomes after maternal Zika virus infection during pregnancy - U.S. territories, January 1, 2016-April 25, 2017. MMWR Morb Mortal Wkly Rep 66: 615-621.

37. Hashimoto Tet al., 2017. Importation of Zika virus from Vietnam to Japan, November 2016. Emerg Infect Dis 23: 1223-1225.

38. Kawai $Y$ et al., 2019. Increased growth ability and pathogenicity of American- and Pacific-subtype Zika virus (ZIKV) strains compared with a Southeast Asian-subtype ZIKV strain. PLoS NegI Trop Dis 13: e0007387.

39. Mulkey SB et al., 2020. Neurodevelopmental abnormalities in children with in utero Zika virus exposure without congenital Zika syndrome. JAMA Pediatr 174: 269-276.

40. Centers for Disease Control and Prevention, 2020. Congenital Zika Syndrome \& Other Birth Defects. Available at: https:// www.cdc.gov/pregnancy/zika/testing-follow-up/zika-syndromebirth-defects.html. Accessed May 12, 2020.

41. Brasil $P$ et al., 2016. Zika virus infection in pregnant women in Rio de Janeiro. N Engl J Med 375: 2321-2334.

42. Honein MA et al., 2017. Birth defects among fetuses and infants of US women with evidence of possible Zika virus infection during pregnancy. JAMA 317: 59-68.

43. Pomar $L$ et al., 2018. Maternal-fetal transmission and adverse perinatal outcomes in pregnant women infected with Zika virus: prospective cohort study in French Guiana. BMJ 363: k4431.

44. Reynolds MR et al., 2017. Vital signs: update on Zika virusassociated birth defects and evaluation of all U.S. infants with congenital Zika virus exposure-U.S. Zika pregnancy registry, 2016. Morb Mortal Wkly Rep 66: 366-373.

45. Rice ME et al., 2018. Vital signs: Zika-associated birth defects and neurodevelopmental abnormalities possibly associated with congenital Zika virus infection-U.S. territories and freely associated states, 2018. Morb Mortal Wkly Rep 67: 858-867.

46. Musso D, Ko Al, Baud D, 2019. Zika virus infection-after the pandemic. N Engl J Med 381: 1444-1457. 